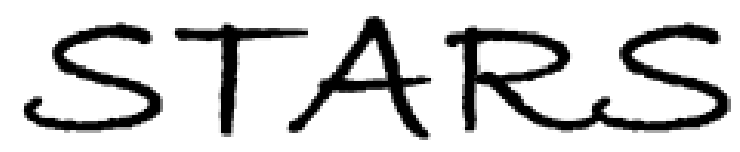

University of Central Florida

STARS

$1-1-2009$

\title{
Encoding and decoding nanoscale thermal barcodes for ultrahigh capacity identification systems
}

\author{
Zeyu Ma \\ University of Central Florida \\ Yan Hong \\ University of Central Florida \\ Minghui Zhang \\ University of Central Florida \\ Ming Su \\ University of Central Florida
}

Find similar works at: https://stars.library.ucf.edu/facultybib2000

University of Central Florida Libraries http://library.ucf.edu

This Article is brought to you for free and open access by the Faculty Bibliography at STARS. It has been accepted for inclusion in Faculty Bibliography 2000 s by an authorized administrator of STARS. For more information, please contact STARS@ucf.edu.

\section{Recommended Citation}

Ma, Zeyu; Hong, Yan; Zhang, Minghui; and Su, Ming, "Encoding and decoding nanoscale thermal barcodes for ultrahigh capacity identification systems" (2009). Faculty Bibliography 2000s. 1853.

https://stars.library.ucf.edu/facultybib2000/1853

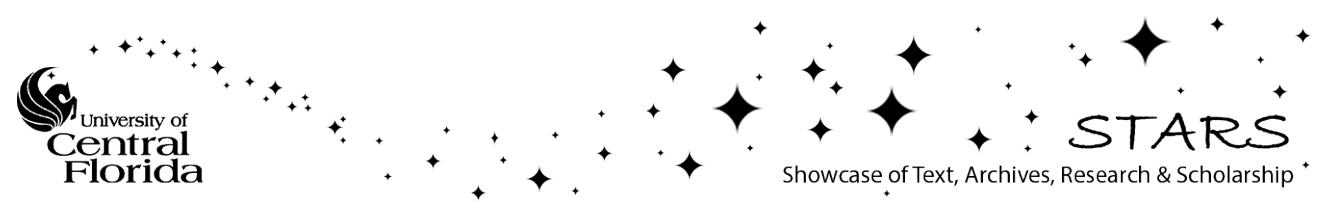




\section{Encoding and decoding nanoscale thermal barcodes for ultrahigh capacity identification systems}

Cite as: Appl. Phys. Lett. 95, 233101 (2009); https://doi.org/10.1063/1.3271524

Submitted: 24 October 2009 . Accepted: 12 November 2009 . Published Online: 07 December 2009

Zeyu Ma, Yan Hong, Minghui Zhang, and Ming Su

\section{ARTICLES YOU MAY BE INTERESTED IN}

Multiplexed highly sensitive detections of cancer biomarkers in thermal space using encapsulated phase change nanoparticles

Applied Physics Letters 95, 043701 (2009); https://doi.org/10.1063/1.3189082

Magnetic NiFe/Au barcode nanowires with self-powered motion

Journal of Applied Physics 111, 07B513 (2012); https://doi.org/10.1063/1.3676062

Synthesis and nanoscale thermal encoding of phase-change nanowires

Applied Physics Letters 90, 183116 (2007); https://doi.org/10.1063/1.2736271

\section{Applied Physics Letters}

Mid-IR and THz frequency combs special collection

\section{Read Now!}




\title{
Encoding and decoding nanoscale thermal barcodes for ultrahigh capacity identification systems
}

\author{
Zeyu Ma, ${ }^{1,2}$ Yan Hong, ${ }^{1,2}$ Minghui Zhang, ${ }^{1,3}$ and Ming Su ${ }^{1,2, a)}$ \\ ${ }^{1}$ NanoScience Technology Center, University of Central Florida, Orlando, Florida 32826, USA \\ ${ }^{2}$ Department of Mechanical, Materials, and Aerospace Engineering, University of Central Florida, Orlando, \\ Florida 32826, USA \\ ${ }^{3}$ Department of Materials Chemistry, College of Chemistry, Nankai University, Tianjin 300071, \\ People's Republic of China
}

(Received 24 October 2009; accepted 12 November 2009; published online 7 December 2009)

\begin{abstract}
An ultrahigh capacity coding system is created based on the solid to liquid phase changes of a variety of nanoparticles, which are designed and prepared with sharp, discrete melting peaks and encapsulated inside nonmelting shells. A mixture of such thermally-active nanoparticles at designed ratio can be embedded inside an object as a nanoscale barcode that can be decoded by differential thermal scan. The coding capacity depends on the number of melting peaks, which in turn depends on nanoparticle compositions. A coding system with 50 different compositions can generate over $10^{15}$ thermal barcodes, sufficient for many identification applications. (c) 2009 American Institute of Physics. [doi:10.1063/1.3271524]
\end{abstract}

The need for an ultrahigh capacity coding system with small dimensions to identify each object of a large group of objects is largely unmet. According to the United States Department of Justice there is over 37\% chance of never finding a suspect in a firearm related crime. ${ }^{1}$ Only $64 \%$ cases of all hit-and-run accidents (5842) in Denver, 2008, have received follow up investigations. If an identification technique could be used to make bullets or paints themselves evidences, the efficiency of law enforcement will be greatly enhanced by having an avenue to solve the most difficult cases. Although widely used to identify commercial products, the existing identification techniques based on numbers (serial number), patterns (optical barcode), materials (microfiber), ${ }^{2}$ and radio frequency devices, ${ }^{3}$ are not appropriate to uniquely encode each object such as bullet or automobile paint. The serial numbers have been engraved on bullets using lasermachining but tangible numbers can be damaged during collisions to lose identification information. The optical barcodes, though convenient to decode, cannot be used in the cases where invisible barcodes are needed to hide identification information. The radio frequency devices are expensive and cannot be miniaturized to identify each object of a large quantity of small objects. These identification techniques require integrity of barcodes; otherwise tracking becomes very difficult or impossible. Microfibers embedded in an object are intangible, and have multiple morphologies but the sizes of microfibers are too big to offer enough coding capacity, and morphological signatures cannot be directly converted to barcodes.

Advances in nanotechnologies have opened the possibility of creating ultrahigh capacity identification system by taking the dimension advantage of nanostructured materials including nanoparticles. Actually, nanoparticles with diameters less than $100 \mathrm{~nm}$ have been made and used as markers to label biomolecules, ${ }^{4,5}$ where the existence of nanoparticles can be detected through their unique physical signatures such as optical, magnetic, electric, or electrochemical properties.

${ }^{\text {a)}}$ Electronic mail: mingsu@mail.ucf.edu.
But the application of nanoparticles to uniquely label each object in a group of objects is limited due to lack of suitable signatures or low level of multiplicity. Although nanoparticles of different kinds can be differentiated by their properties, the existing categories of physical properties are limited, and identifying nanoparticles based on the same signature would be very challenging, if not impossible. One type of magnetic (conductive) nanoparticle cannot be uniquely distinguished from other type of magnetic (conductive) nanoparticles by according measurement. Metallic and semiconductor nanoparticles have size-dependent optical properties $^{6,7}$ but the absorbance peaks in the convenient ultraviolet-visible region are broad with peak width at half height of more than $150 \mathrm{~nm}$, which limits the types of optically detectable nanoparticles (i.e., multiplicity) to a few in the wavelength range from 400 to $900 \mathrm{~nm}$. Most important, these methods require sophisticated readout devices, and the detection signals can be are significantly suppressed or interfered by the surrounding environments or matrices.

We have developed an ultrahigh capacity thermal encoding-decoding technique, which is based on the solidliquid phase changes of nanoparticles and can be embedded in target objects. The thermal signal transduction mechanism has no macroscopic analog but brings long-pursuing multiplicity to the area of nanotechnology by taking the advantage of a well-known phenomenon as follows: the temperature of a single component solid does not rise above its melting temperature until the entire solid is molten, which suggests the theoretical width of the melting peak will be zero as the temperature increases linearly. A series of nanoparticles with sharp, discrete melting peaks can be designed. The sharp peak over a large temperature range means the multiplicity of thermal coding method can be huge, providing sufficient types of nanoparticles can be made with desired melting points. A combination of nanoparticles with different melting temperatures is encoded and embedded in an object as barcode. Other than sharp melting peaks and large thermal scan range, the thermal barcodes can be generated by mixing nanoparticles of different melting temperatures, dispersed 
uniformly inside an object without changing its properties. The phase change nanoparticles cannot be removed or changed once dispersed, and can be decoded even if only part of object is available.

Assuming that each melting peak is sufficiently sharp and does not overlap each other, a one to one correspondence between melting peak and nanoparticles composition can be created. The encoding capacity is dependent on the number of melting peak or nanoparticle composition, and the number of different barcodes can be derived from the total number of peaks using

$$
\sum_{k=1}^{n} C_{n}^{k} \approx \sum_{k=1}^{n} \frac{n !}{k !(n-k) !}=2^{n},
$$

where $n$ and $k$ are the total number of melting peaks, and the number of melting peaks in one combination, respectively. From the equation, ten different nanoparticles can produce more than 1000 barcodes; 50 different nanoparticles are able to generate more than $10^{15}$ different barcodes, which is sufficiently large for most of the identification requirements. Several unanswered questions are (1) how to make 50 different types of nanoparticles with discrete melting peaks, (2) how to avoid signal interference among nanoparticles and with matrix, and (3) how to incorporate and decode such nanoscale thermal barcodes. In the following sections, we will demonstrate the feasibility of making nanoparticles of low melting point metals and alloys, encoding barcodes by mixing nanoparticles at predesigned combinations and ratios, and decoding barcodes by using differential thermal analysis.

The low melting metallic nanoparticles are produced at large scale by boiling powders of appropriate metals or alloys in high boiling temperature fluids such as polyalphaolefin (PAO) or ethylene glycol (EG). ${ }^{8,9}$ For example, $0.1 \mathrm{~g}$ of indium powders $(-325 \mathrm{mesh})$ is heated at $200{ }^{\circ} \mathrm{C}$ in $20 \mathrm{ml}$ of PAO under nitrogen protection for $2 \mathrm{~h}$ to make indium nanoparticles. Similar method is used to make bismuth and tin nanoparticles. The alloy nanoparticles are made through a two-step process that involves making alloy and boiling to nanoparticles. The as-made nanoparticles are encapsulated in high melting point silica shells by thermal decomposition of tetraethylorthosilicate. ${ }^{10}$ This method is able to make encapsulated nanoparticles with core diameter of about $100 \mathrm{~nm}$ and shell thickness of 10-20 nm. The morphologies, structures, and compositions of the nanoparticles have been confirmed by transmission electron microscopy (TEM), selected area electron diffraction (SAED), and energy dispersive $\mathrm{x}$-ray analysis (EDX). The diffraction patterns at room temperature reflect the crystalline structures, and EDX confirms coexistence of lead and tin in alloy nanoparticles. Although the number of pure metals or binary eutectic alloys with melting temperature lower than $300{ }^{\circ} \mathrm{C}$ is limited, adding a third metal is one way to reduce the melting temperature of binary eutectic alloy, and increasing the temperature range up to $1000{ }^{\circ} \mathrm{C}$ could be another solution. We have made ternary alloy nanoparticles with melting temperatures of $181{ }^{\circ} \mathrm{C}\left(\mathrm{Sn}_{63} \mathrm{~Pb}_{35} \mathrm{Ag}_{2}\right) .^{11}$

Taking four types of nanoparticles (indium, lead-tin alloy, tin, and bismuth) as examples, encoding can be carried out by incorporating a homogeneous mixture of nanoparticles in objects; decoding can be carried out by measuring the thermal properties of an object or a piece of object. The melting temperatures of nanoparticles of indium, lead-tin al-

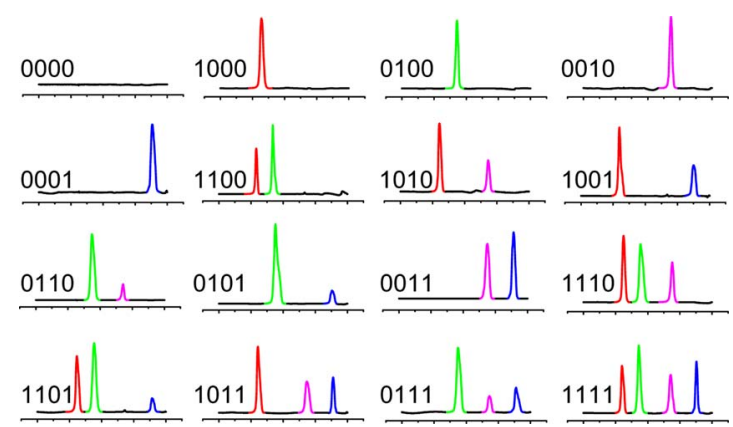

FIG. 1. (Color online) DSC curves of nanoscale thermal barcodes, where the scale range from left to right is from 100 to $300{ }^{\circ} \mathrm{C}$.

loy, tin, and bismuth have been determined to be 156,183 , 232, and $271{ }^{\circ} \mathrm{C}$ using heat flux different scanning calorimetry (DSC) (PerkinElmer DSC7). The areas of melting peaks (i.e., heat fluxes) are proportional to the masses and the latent heats of fusion of the materials. In the four element system, each melting peak can be denoted as one or zero depending on whether the heat flux is higher or lower than a threshold value. The 16 combinations of four elements are 0000,1000 , 0100, 0010, 0001, 1100, 1010, 1001, 0110, 0101, 0011, 0111, 1001, 1101, 1011, and 1111. The nanoparticles are simply mixed with different mass ratio to produce a homogeneous mixture. The information in each mixture is decoded by adding $5 \mathrm{mg}$ of a mixture into an aluminum pan and counting the melting peaks in temperature rise process. Figure 1 shows the DSC curves collected from 16 different mixtures of nanoparticles, where each curve can be repeated for many times in the same scan range, meaning the oxidation of nanoparticles is not an issue. Each DSC curve is flatten to remove its slope and smoothened to remove thermal fluctuation. The time it takes to scan from 20 to $300{ }^{\circ} \mathrm{C}$ at ramp rate of $10{ }^{\circ} \mathrm{C}$ per minute is $28 \mathrm{~min}$, and the decoding time could be reduced substantially by increasing ramp rates. In one case, where four peaks are scanned at $20{ }^{\circ} \mathrm{C}$ per minute, and the total scan time is about 14 min.

Figure 2(a) shows the widths of melting peaks are dependent on the ramp rates of thermal measurements and can be smaller than $0.7^{\circ} \mathrm{C}$ at the ramp rate of $1{ }^{\circ} \mathrm{C}$ per minute. The sensitivity of decoding is dependent on the minimal heat flux that can be measured by DSC, and the lowest concentration of phase change materials in an available sample. The root-mean-square sensitivity of DSC is about $0.02 \mu \mathrm{W}$, which corresponds to $0.05 \mathrm{mg}$ bismuth at ramp rate of $2{ }^{\circ} \mathrm{C}$ per second. In order to produce detectable heat flow, the concentration of nanoparticles in each object should be sufficient, providing the mass of the object is constant. On the other hand, the nanoparticles should not change the properties of the object dramatically. In one example, $10 \mathrm{mg}$ of
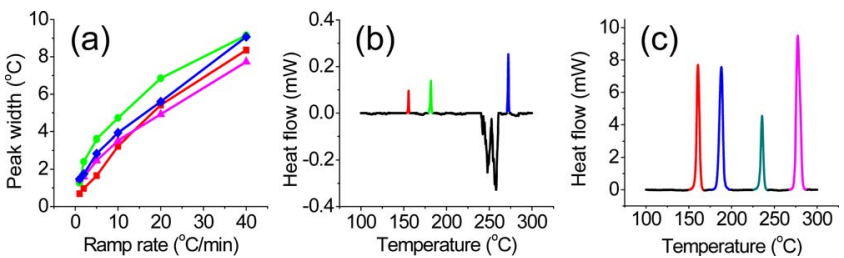

FIG. 2. (Color online) Ramp rate dependent peak width of indium (square), tin (triangle), lead-tin (circle), and bismuth (diamond) nanoparticles (a). DSC curves of two thermal barcodes embedded in paint (b) and aluminum (c) 
mixture of nanoparticles is dispersed in a commercial paint (Glidden green paint G31). Due to the small amount of nanoparticles, the color of paint after solidification is the same. A small piece of paint is knocked down and decoded by using DSC. Figure 2(b) shows the DSC curves of paint with barcode, where three melting peaks are identified from the DSC curves and decoded as 1101. In comparison, the DSC curve of paint only shows exothermic peaks generated from its burning process. The barcodes can be embedded in aluminum by sintering aluminum powders and a combination of nanoparticles at $800{ }^{\circ} \mathrm{C}$ in vacuum. Figure 2(c) shows the DSC curves of aluminum with barcodes, where the melting peaks are decoded as 1111 . The aluminum melts at $660{ }^{\circ} \mathrm{C}$ and does not interfere with the thermal signatures. In the case that the melting peak of the matrix overlaps with those of nanoparticles, the overall peak can be deconvoluted to exclude the possible interference.

The long term stability of nanoparticles at room temperature has been confirmed by the identical melting behaviors of nanoparticles after one year. The thermal stability of nanobarcode has been studied by heating nanoparticles to high temperature. The silica shells prevent leakages and coalescences of molten materials in the range from 20 to $700{ }^{\circ} \mathrm{C}$, and simplify the decoding process, especially when the thermal decoding should be repeated for few times. We have heated encapsulated lead-bismuth alloy nanoparticles inside a TEM. Even if the temperature is higher than the melting temperature of the alloy $\left(182{ }^{\circ} \mathrm{C}\right)$, the nanoparticles do not show morphological changes (not shown). The changes in diffraction patterns confirm the solid liquid phase change of encapsulated nanoparticles (not shown). The silica shell breaks as the temperature is increased to $650{ }^{\circ} \mathrm{C}$. Figures 3(a) and 3(b) are two images collected from the same area with 2 min interval, where the arrows indicate the leakage of core material. The damage to shells could be induced by large unbalanced vapor pressures of metal atoms inside

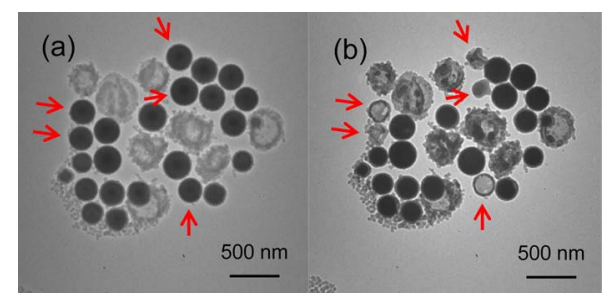

FIG. 3. (Color online) TEM images of silica encapsulated lead-bismuth nanoparticles at $650{ }^{\circ} \mathrm{C}$ collected with 2 min interval, where (a) is collected earlier than (b).

and outside the shell. As long as the temperature is less than $500{ }^{\circ} \mathrm{C}$, the silica shells protect metal cores from leakage for a long time, and the thermal behaviors can be repeatable for many times.

This nanoparticle synthesis part of the work has been supported by Air Force Research Laboratory and National Sciences Foundation (Grant No. CBET 0828466).

${ }^{1}$ R. G. Atchison, U.S. Patent No. 5,685,100 (11 November 1997).

${ }^{2}$ S. R. Nicewarner-Pena, R. G. Freeman, B. D. Reiss, L. He, D. J. Pena, I. D. Walton, R. Cromer, C. D. Keating, and M. J. Natan, Science 294, 137 (2001)

${ }^{3}$ S. Sarma, D. Brock, and D. Engels, IEEE MICRO 21, 50 (2001).

${ }^{4}$ P. Alivisatos, Nat. Biotechnol. 22, 47 (2004).

${ }^{5}$ N. L. Rosi and C. A. Mirkin, Chem. Rev. (Washington, D. C.) 105,1547 (2005)

${ }^{6}$ R. D. Averitt, S. L. Westcott, and M. J. Halas, J. Opt. Soc. Am. B 16, 1824 (1999).

${ }^{7}$ R. Lopez, L. C. Feldman, and R. F. Haglund, Phys. Rev. Lett. 93, 177403 (2004).

${ }^{8}$ L. Ma, Y. Hong, Z. Ma, C. Kaittanis, J. M. Perez, and M. Su, Appl. Phys. Lett. 95, 043701 (2009).

${ }^{9}$ Y. Wang and Y. Xia, Nano Lett. 4, 2047 (2004).

${ }^{10}$ C. Graf, D. L. J. Vossen, A. Imhof, and A. V. Blaaderen, Langmuir 19, $6693(2003)$

${ }^{11}$ C. D. Zou, Y. L. Gao, B. Yang, X. Z. Xia, Q. J. Zhai, C. Andersson, and J. Liu, J. Electron. Mater. 38, 351 (2009). 